\title{
Desigualdades en la mortalidad por enfermedades cardiovasculares en los municipios del Eje Cafetero, 2009-2011
}

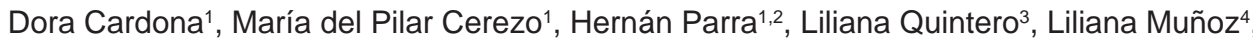 \\ Olga Lucía Cifuentes ${ }^{1}$, Silvia Clemencia Vélez ${ }^{3}$ \\ 1 Grupo de Investigación en Salud Pública, Universidad Autónoma de Manizales, Manizales, Colombia
2 Facultad de Ciencias Exactas y Naturales, Universidad Nacional de Colombia, Manizales, Colombia
${ }^{3}$ Secretaría de Salud de Armenia, Armenia, Colombia
${ }^{4}$ Grupo de Investigación en Epidemiología, Violencia y Sociedad, Fundación Universitaria del Área Andina,
Pereira, Colombia \\ Institución donde se llevó a cabo el estudio: Universidad Autónoma de Manizales
}

Introducción. El impacto de la mortalidad por enfermedades cardiovasculares requiere medir la relación entre las condiciones socioeconómicas locales y estas causas de muerte.

Objetivo. Determinar la desigualdad en la mortalidad por enfermedades cardiovasculares en los municipios del Eje Cafetero (2009-2011).

Materiales y métodos. Se hizo un estudio ecológico en el que se comparó la mortalidad por enfermedades cardiovasculares (hipertensivas, isquémicas, cerebrovasculares) en los municipios con base en su situación económica. Los datos de mortalidad y el índice de necesidades básicas insatisfechas se obtuvieron de las estadísticas vitales del Departamento Administrativo Nacional de Estadística (DANE), en tanto que el producto interno bruto municipal per cápita se calculó para el estudio. Los índices de desigualdad empleados se calcularon por rangos y en modelos de regresión, así como con los índices de concentración, y de Theil, utilizando el programa Epidat 3.1.

Resultados. El riesgo de morir por enfermedad isquémica e hipertensiva resultó mayor en los municipios con el mayor índice de necesidades básicas insatisfechas. La mortalidad por enfermedad hipertensiva también tendió a concentrarse en dichos municipios. Se encontraron más muertes por enfermedad hipertensiva en los municipios con menor producto interno bruto per cápita en 2009 y 2010, y por enfermedad isquémica, en 2010 y 2011. No obstante, este indicador no mide la brecha entre las comunidades pobres.

Conclusiones. Se carece de indicadores de desigualdad desagregados a nivel de municipio. Los sugeridos con este propósito se calculan para el nivel nacional y departamental, lo que no favorece la caracterización de las desigualdades sociales en salud a nivel territorial.

Palabras clave: enfermedades cardiovasculares, isquemia miocárdica, accidente cerebrovascular, desigualdades en la salud, necesidades y demandas de servicios de salud, producto interno bruto.

doi: http://dx.doi.org/10.7705/biomedica.v35i3.2588

Inequalities in mortality by cardiovascular diseases in the Colombian Coffee Growing Region, 2009-2011

Introduction: The impact of mortality from cardiovascular diseases requires the measurement of the relationship between the local socioeconomic conditions and these death causes.

Objective: To determine the inequality in mortality from cardiovascular diseases in the municipalities of the Colombian Coffee Growing Region (2009-2011).

Materials and methods: We conducted an ecological study to compare the mortality from cardiovascular diseases (hypertensive, ischemic, cerebrovascular) in municipalities and their economic situation. Mortality rates and the index of unsatisfied basic needs were obtained from the Colombian Departamento Administrativo Nacional de Estadística (DANE) vital statistics, while the municipal gross domestic product per capita was estimated for this study. The inequality indices were calculated using regression models, and concentration and Theil indices with Epidat 3.1.

Results: The death risk resulting from ischemic or hypertensive diseases was greater in those municipalities with a higher index of unsatisfied basic needs. Mortality due to hypertensive disease

\section{Contribución de los autores:}

Dora Cardona y Hernán Parra: formulación y evaluación del proyecto

María del Pilar Cerezo y Olga Lucia Cifuentes: formulación del proyecto

Todos los autores participaron en la elaboración del manuscrito. 
tended to concentrate in municipalities with a higher level of unsatisfied basic needs. The municipalities with a lower gross domestic product showed a higher rate of deaths due to hypertensive disease in years 2009 and 2010, and due to ischemic disease in years 2010 and 2011. Nevertheless, this indicator does not measure the gap existing among poor communities.

Conclusions: Disaggregated inequality indicators at municipal level are lacking. Suggested indicators are estimated only for country and provincial levels and they do not favor the characterization of health social inequalities at territorial level.

Key words: Cardiovascular disease, myocardial ischemia, stroke, health inequalities, health services needs and demand, gross domestic product.

doi: http://dx.doi.org/10.7705/biomedica.v35i3.2588

En el perfil de la mortalidad en América Latina y el Caribe para el 2001, se señala que las enfermedades isquémicas del corazón son responsables de $10,9 \%$ de las defunciones, seguidas de las enfermedades cerebrovasculares $(8,2 \%)$, la diabetes mellitus $(5,0 \%)$, las infecciones respiratorias $(4,8 \%)$, las enfermedades respiratorias crónicas $(3,0 \%)$, y la hipertensión arterial sistémica $(2,7 \%)(1)$.

Entre 2005 y 2010, las enfermedades cardiovasculares fueron la principal causa de muerte en la gran mayoría de los países de la región. El 30 $\%$ de las muertes prematuras por enfermedades cardiovasculares ocurrió en el quintil más pobre, mientras que solo el $13 \%$ correspondió al quintil más rico (2). Según el Análisis de Situación de Salud (ASIS) de 2010, las principales causas de muerte entre 1997 y 2010 en Colombia, fueron las enfermedades isquémicas $(45,7 \%)$ y las cerebrovasculares $(23,8 \%)$ (3). Cabe resaltar que el centro del país concentró el $50,4 \%$ de estas muertes (4).

Según el informe del Boletín № 1 del Observatorio Nacional de Salud, 2013, en los departamentos de Caldas, Quindío y Risaralda se presentaron las tasas más altas de mortalidad por enfermedades cardiovasculares, todas superiores al promedio nacional (5).

Además, el mayor porcentaje de la carga de enfermedad estimada según los años de vida saludables perdidos por discapacidad o muerte prematura, correspondió a las enfermedades crónicas no transmisibles (76 \%) (6).

\footnotetext{
Correspondencia:

Dora Cardona, Grupo de Investigación en Salud Pública, Universidad Autónoma de Manizales, Antigua Estación del Ferrocarril, Manizales, Colombia. Teléfono: (576) 8727272 , extensión 166 dcrivas@autonoma.edu.co

Recibido: 21/11/14; aceptado: 29/04/15
}

Las orientaciones del Estado dirigidas a resolver la problemática de las enfermedades en general, y de las crónicas en particular, se explicitan en el Plan Nacional de Salud Pública, 2007-2010, concretamente en los componentes de la línea de vigilancia en salud pública, la cual pone el énfasis en los riesgos y daños biológicos, del comportamiento y del medio ambiente tanto individuales como colectivos. La norma incluye, además de la notificación obligatoria, la investigación de los eventos priorizados con base en modelos de riesgo y enfermedad, con el objetivo de disminuir las enfermedades crónicas no transmisibles y las discapacidades (7). Entre las estrategias de dicho plan se propone: desarrollar modelos y formas de atención diferencial sostenibles para las enfermedades no transmisibles; implantar procesos para la vigilancia y el control; evaluar e investigar los determinantes sociales de la salud, y otras acciones relacionadas con las condiciones crónicas prevalentes.

La Organización Mundial de la Salud (OMS) en su informe global sobre las enfermedades crónicas no transmisibles, y la Asamblea General de las Naciones Unidas en una declaración recientemente adoptada en torno a estas, han hecho un llamado a la comunidad mundial para que se reconozcan las repercusiones devastadoras de las enfermedades no transmisibles en todo el mundo y la importancia de adoptar medidas encaminadas a reducirlas (8). Otras directrices internacionales, como la declaración ministerial para la prevención y control de las enfermedades crónicas adoptada en México en 2011 y la estrategia para la prevención y el control de las enfermedades crónicas no transmisibles, 2012-2025, aprobada en la 28 ${ }^{\text {a }}$ Conferencia Sanitaria Panamericana, reiteran el compromiso de los países con la formulación de estrategias para el control de dichas enfermedades (9).

Al citar la declaración ministerial para la prevención y control de las enfermedades crónicas de 2011, la OMS señaló en el 2012 que las políticas públicas 
para combatir las enfermedades no transmisibles también deben tener en cuenta determinantes sociales como la equidad, el sexo, la educación, los derechos humanos y la protección social, ya que existen grandes desigualdades en cuanto a la situación de salud y el acceso a los servicios de atención.

Los factores determinantes sociales de la salud reflejan las condiciones socioeconómicas en las que transcurre la vida de las poblaciones, así como su ubicación social y las posibilidades de acceso al ingreso, la educación y los demás componentes del bienestar; con ellos se constatan las diferencias en los resultados de salud, que pueden constituirse en desigualdades o en 'inequidades'.

Desde su creación en 2005, la Comisión sobre Determinantes Sociales de la Salud de la OMS ha señalado que la gestión sanitaria debe aclarar la relación entre los factores determinantes sociales y las desigualdades, así como las relaciones entre dichos factores, para darle factibilidad a la proyección de las políticas en salud (10). Las acciones encaminadas a modificar los determinantes sociales se fundamentan en la reducción de las inequidades en salud, y en el mejoramiento de la salud y el bienestar, estableciendo las prioridades pertinentes (11).

En Colombia, los primeros informes sobre desigualdades en salud se hicieron a partir de los estudios nacionales de salud, cuyos análisis se hacían por regiones, con énfasis en la exposición diferencial de las poblaciones a factores conocidos de riesgo. En el marco de los factores determinantes sociales, dicho análisis da cuenta de la situación de la salud, diferenciando conceptualmente las desigualdades de las inequidades. Las primeras se refieren a las diferencias en la manifestación de un problema sanitario respecto de algún factor asociado, sin que en su medición se tengan en cuenta consideraciones relativas a la justicia. Las segundas, es decir, las inequidades, aluden a las diferencias "que se consideran injustas, injustificadas, evitables e innecesarias, y que sistemáticamente agobian a las poblaciones que se han vuelto vulnerables a causa de estructuras sociales fundamentales y de instituciones políticas, económicas y jurídicas que prevalecen, tales como las diferencias relacionadas con el género, el ingreso, el nivel educativo, la posición ocupacional, el estrato social y el nivel de desarrollo de las regiones" (12).

El Plan Decenal de Salud (2014-2021) introduce en la agenda pública de Colombia la perspectiva de los factores determinantes sociales de la salud y las desigualdades en este campo, como horizonte para la planeación, el seguimiento y la evaluación en los territorios. En este sentido, cobra relevancia la caracterización de la morbilidad y la mortalidad por enfermedades cardiovasculares a nivel territorial y su relación con los indicadores económicos de medición de la pobreza que subyacen a las variables identificadas relacionadas con la desigualdad.

Según Caballero (12), para medir la pobreza se han utilizado en el país instrumentos como los indicadores antropométricos, el producto interno bruto (PIB), el índice de necesidades básicas insatisfechas, la línea de indigencia y la línea de pobreza, el método integrado de medición de la pobreza, la estratificación, el índice de condiciones de vida, el Sisbén (Sistema de Identificación y Clasificación de Potenciales Beneficiarios para Programas Sociales), el índice de desarrollo humano, la pobreza subjetiva y el índice multidimensional de la pobreza. En cuanto a la medición de la desigualdad, se recurre al índice de Gini, el índice de desarrollo humano ajustado por desigualdad y el índice de sexo, de los cuales el índice de desarrollo humano ajustado por desigualdad es el que incluye la salud.

Sin embargo, solo unos pocos de los indicadores mencionados están disponibles a nivel local; es el caso del PIB per cápita y del índice de necesidades básicas insatisfechas. EI PIB per cápita se calcula como la relación entre el valor de producción de bienes y servicios de la economía, y la población total de un país. Debido a que se basa en el ingreso promedio de la población, es un indicador poco eficiente de la pobreza, aunque su capacidad de revelar la asociación del ingreso per cápita con los mayores niveles de desarrollo encontrados y otros indicadores de pobreza, además de su disponibilidad año tras año, facilitan la comparación y el análisis continuo.

En América Latina, el uso del índice de necesidades básicas insatisfechas, que no es un método de medición de la pobreza propiamente, ha significado un aporte importante en la identificación de ciertas carencias críticas de la población que tienen relación con la pobreza (13). La medición de las necesidades básicas insatisfechas en Colombia toma en cuenta las siguientes condiciones observables: viviendas con hacinamiento crítico, condiciones físicas impropias para el alojamiento humano, servicios inadecuados, gran dependencia económica y niños en edad escolar que no asisten a la escuela. 
Para la medición de los niveles de salud, en la literatura especializada se sugiere recurrir a los datos de la mortalidad general, la mortalidad por causas específicas, la salud percibida, la utilización de los servicios de salud y la prevalencia de enfermedades.

El objetivo de este estudio fue medir las desigualdades en la mortalidad por enfermedades hipertensivas, isquémicas y cerebrovasculares, en los municipios del Eje Cafetero, mediante la comparación de las tasas de mortalidad específicas, los valores del PIB per cápita y el nivel de necesidades básicas insatisfechas en cada municipio mediante técnicas propuestas por varios autores (14).

\section{Materiales y métodos}

Se hizo un estudio ecológico para medir las desigualdades sociales con base en el PIB, el índice de necesidades básicas insatisfechas y la mortalidad por enfermedades cardiovasculares en 53 municipios del llamado Triángulo del Café, ubicados en los departamentos de Caldas, Quindío y Risaralda.

Las fuentes de información del estudio fueron secundarias. Las causas de mortalidad se obtuvieron de los registros oficiales de defunción del DANE (muertes no fetales); de esta misma entidad se obtuvieron los datos sobre necesidades básicas insatisfechas a nivel municipal. Para el cálculo del producto interno bruto de los municipios, se utilizó la información sobre las ramas de actividad económica en cada uno de ellos. Con base en el valor de este producto se calcularon los PIB per cápita por municipio.

Como variables dependientes se tomaron las tasas de mortalidad por enfermedades hipertensivas, isquémicas y cerebrovasculares, con base en la causa básica de muerte codificada según la CIE10 y registrada por el DANE. Como variables independientes se utilizaron el PIB per cápita calculado específicamente para el estudio y el índice de necesidades básicas insatisfechas obtenido del DANE (15).

\section{Obtención y transformación de la información}

Ajuste de tasas. Para hacer comparables los análisis de la mortalidad en los diferentes municipios cubiertos por el estudio, las tasas brutas se ajustaron por grupos de edad utilizando como estándar los datos del censo del DANE del 2005. Las poblaciones se estratificaron por grupos de edad, así: 0 a 44 años; 45 a 64 años, y 65 años y más. Las cifras de población estándar fueron las siguientes: en el grupo de 0 a 44 años, 33'365.865 personas; en el grupo de 45 a 65 años, 6'840.147, y en el grupo de 65 y más, 2'682.580, para un total de 40'206.012.

El ajuste se hizo con el método directo, de la siguiente manera: para cada año y localidad, las tasas específicas de mortalidad de cada estrato se estimaron dividiendo el número de muertes por su respectiva población. La tasa específica observada en cada estrato se multiplicó por el tamaño de la población estándar correspondiente, y la suma del número de defunciones ajustadas por las poblaciones de los estratos se dividió por el total de la población estándar, con el fin de obtener tasas ajustadas comparables.

En los municipios con registros faltantes de defunción, se optó por atribuirles promedios con la información disponible en cada uno de ellos. Estos municipios fueron los siguientes: Pueblo Rico, Marulanda, Norcasia, Pácora, Buenavista, Calarcá, Balboa, Córdoba y Santuario. En este proceso se excluyó al municipio de Belalcázar y en el análisis de la mortalidad por enfermedades hipertensivas se excluyó a Salento, debido a la ausencia de información.

\section{Producto interno bruto}

Con base en la información disponible a nivel municipal, el PIB se calculó sumando el valor agregado bruto (VAB) de cada rama de actividad económica $\left(V A B_{i}\right)$, lo que equivale al valor de los bienes y servicios finales, más los impuestos netos de subsidios sobre la producción y las importaciones $\left(T_{x s}\right)$. Se llama valor agregado bruto porque no se le ha descontado el valor de la depreciación. Este cálculo se puede expresar con la siguiente ecuación.

$$
P I B=\sum_{i}^{n} V A B i+T_{x s}
$$

Las ramas de la actividad económica sumaron 31 (agrícola sin café, café, industria, comercio, etc.) y se dividieron en 16 grupos (actividad agropecuaria, industria, construcción, etc.).

La medición del PIB municipal se hizo siguiendo la última versión del sistema de cuentas nacionales, basada en el sistema recomendado por la Comisión Europea según lo reporta Vallecilla (16).

Las series del PIB se presentan en precios corrientes y constantes de 2005 (PIB real), año base vigente para las cuentas nacionales en Colombia. 
EI PIB per cápita corresponde a la suma del valor de bienes y servicios finales (valor agregado) de las diferentes ramas de la actividad económica (agricultura, industria, transporte, etc.), más los impuestos a las importaciones, más el IVA no deducible, más el impuesto sobre el producto, menos subsidios, dividido por el tamaño de la población. Para el estudio, los datos del PIB per cápita se obtuvieron del PIB municipal calculado y las proyecciones oficiales de población por municipio estimadas por el DANE.

\section{Necesidades básicas insatisfechas}

El índice de necesidades básicas insatisfechas es un indicador de las condiciones de bienestar material de la población, y para calcularlo se toma como unidad de observación la vivienda y las personas que residen en ella.

Con esta metodología se busca determinar, con ayuda de algunos indicadores simples, si las necesidades básicas de la población se encuentran cubiertas. Los grupos que no alcancen un umbral mínimo fijado, se clasifican como pobres. Los indicadores seleccionados en este caso fueron: las viviendas inadecuadas, las viviendas con hacinamiento crítico, las viviendas con servicios inadecuados, las viviendas con alta dependencia económica y las viviendas con niños en edad escolar que no asistían a la escuela.

El índice de necesidades básicas insatisfechas es un indicador directo de carencias críticas en una población y, en tal sentido, un indicador de pobreza relativamente estable a lo largo del tiempo. Para este estudio, los valores de necesidades básicas insatisfechas por municipio se obtuvieron del DANE.

\section{Métodos de análisis}

Se describió el comportamiento de las variables dependientes e independientes en los 53 municipios mediante medidas de posición, y se calcularon los promedios y la desviación de las variables agrupadas por cuartiles. Los municipios se ordenaron según las variables socioeconómicas para proceder a su comparación.

El análisis de las desigualdades se hizo con el programa Epidat 4.0 (17), el cual se utiliza en los análisis de la situación de salud en el país. Con la medición de las desigualdades sociales se compara el estado de salud de dos o más grupos de población estratificados según su condición socioeconómica. Dependiendo de las medidas elegidas, el resultado cuantitativo puede expresarse en términos absolutos o relativos.
Para el análisis se utilizaron las siguientes mediciones de desigualdad.

Medidas de desigualdad basadas en rangos. Estas mediciones comparan un mismo indicador de salud (por ejemplo, la mortalidad) en las dos poblaciones de la serie analizada que tengan condiciones socioeconómicas extremas: la mejor y la peor. La comparación del indicador de salud entre ellas puede hacerse mediante división (cociente) o resta, es decir, la razón (cociente) de tasas extremas, o la diferencia de tasas extremas. Ambas pueden ser útiles, aunque los cocientes son más fáciles de interpretar como riesgos relativos.

Medidas de desproporcionalidad. Se basan en el índice de concentración y la curva de concentración. En el caso de la salud, estos análisis se usan para dar una medida conjunta de las desigualdades en varias localidades. Lo que se hace mediante estas técnicas es distribuir equitativamente los valores de una variable ordenados de menor a mayor entre las poblaciones, las cuales también se ordenan según sus condiciones socioeconómicas, asumiendo teóricamente que el indicador se reparte por igual entre ellas. Esta distribución uniforme se representa en el plano cartesiano como una línea diagonal cuya pendiente es 1 y cuyo intercepto es 0 . La diagonal representaría una igualdad teórica simétrica y perfecta en la distribución de los valores, y se usa como estándar para valorar los datos reales observados. Cuando los datos reales se llevan al plano, forman una curva que puede coincidir o no con la diagonal. Mientras más desigualdades haya en la distribución, más se alejarán los datos de la diagonal. Esta distancia o dispersión frente a la diagonal puede evaluarse visualmente, pero también puede medirse mediante índices o coeficientes de concentración que oscilan entre -1 y +1 ; un índice de concentración de cero significa igualdad perfecta; mientras más se distancie el decimal hacia el -1 o el +1 , mayor desigualdad habrá en el grupo. Las mediciones de desproporcionalidad más usadas son la curva de Lorenz y el índice de Gini.

Medidas basadas en la regresión. Con estas se calcula una ecuación de regresión en la que el indicador de morbilidad o mortalidad es la variable dependiente $\mathrm{Y}$, en tanto que la variable socioeconómica es la variable explicativa. Las regresiones pueden ser lineales o logísticas, dependiendo de la naturaleza cuantitativa o cualitativa del indicador de morbilidad o mortalidad. Lo usual es armar los modelos de regresión comparando 
el riesgo de enfermar o morir de los grupos en peores condiciones con el de los grupos en mejores condiciones. De este modo, los análisis de regresión permiten calcular dos mediciones de la desigualdad: el índice relativo de desigualdad (IRD), que refleja el número de veces que la morbilidad o la mortalidad es más alta en el extremo con mayor privación económica (bajo PIB o alta proporción de población con necesidades básicas insatisfechas), y el índice relativo de desigualdad acotado (IRDA), que revela el cociente esperado de las tasas extremas de acuerdo con el modelo teórico de la desigualdad asumido para elaborar la ecuación de regresión.

Índice basado en el concepto de entropía (índice de Theil). Estos análisis se basan en la identificación de la proporción de la población que presenta la mayor parte de la enfermedad o la mortalidad, con el fin de asignarle esta carga. El resultado del índice indica la proporción de la población que soporta la carga de enfermedad y permite calcular la proporción de dicha carga que debe redistribuirse para lograr una distribución similar del problema entre los municipios.

\section{Consideraciones éticas}

Según los enunciados del Artículo 11, Capítulo I, de la Resolución número 8430 de 1993, el estudio correspondió a una investigación sin riesgo. A esta categoría corresponden los estudios que emplean técnicas y métodos de investigación documental retrospectivos y en los que no se realiza intervención o modificación intencionada de las variables de los individuos que participan en el estudio. La investigación se hizo con base en los registros de mortalidad codificados según la CIE-10, utilizados en las estadísticas vitales del DANE.

\section{Resultados}

Los resultados del estudio se presentan en el siguiente orden: comportamiento del PIB per cápita, de las necesidades básicas insatisfechas, de las tasas de mortalidad por enfermedades cardiovasculares y, por último, de las desigualdades en la mortalidad.

\section{Comportamiento del PIB per cápita y de las necesidades básicas insatisfechas}

En el cuadro 1 se presentan los valores promedio y la desviación del PIB per cápita por cuartiles de los 53 municipios del Eje Cafetero en los años 2009, 2010 y 2011.

En el 2009, se ubicaron en el cuartil del menor PIB per cápita 13 municipios, de los cuales diez (10/27) pertenecen al departamento de Caldas y tres al departamento de Risaralda (3/14). La distribución varió muy poco en 2010 y 2011.

En el departamento de Risaralda, Quinchía fue el municipio con menor PIB per cápita en el trienio, con una disminución del PIB per cápita entre 0,10 y $012 \%$ entre 2009 y 2010.

Los cinco municipios con menor PIB per cápita derivaban su economía de la agricultura, especialmente del café y la panela. De estos, Pensilvania (Caldas) no registró aumento en el PIB municipal durante el período observado, lo cual incidió negativamente en el valor del PIB per cápita.

Los municipios con mayor PIB per cápita en el Eje Cafetero en el 2009 fueron cuatro del departamento de Caldas, cinco de Risaralda y tres del Quindío. En seis municipios se registraron los valores más altos del PIB per cápita durante los tres años: Balboa (Risaralda), Buenavista (Quindío), Marmato (Caldas), Manizales (Caldas), Filandia (Quindío), Pereira (Risaralda) y Palestina (Caldas).

En los tres primeros municipios mencionados se reflejó la riqueza producida por la explotación de la caña de azúcar en Balboa, la caficultura en Buenavista y la minería en Marmato. Con excepción de estos municipios, que reúnen características especiales, el PIB departamental se concentró en las áreas metropolitanas o ciudades capitales y en los municipios aledaños, donde se

Cuadro 1. Promedio y desviación del PIB per cápita (en millones de pesos) en 53 municipios del Eje Cafetero, 2009-2011

\begin{tabular}{lcccccc}
\hline & \multicolumn{2}{c}{2009} & \multicolumn{2}{c}{$\mathbf{2 0 1 0}$} & \multicolumn{2}{c}{$\mathbf{2 0 1 1}$} \\
\cline { 2 - 7 } Cuartiles & Promedio & Desviación & Promedio & Desviación & Promedio & Desviación \\
\hline Q1 & 4,23 & 0,357788 & 4,32 & 0,367643 & 4,34 & 0,300188 \\
Q2 & 5,17 & 0,246214 & 5,31 & 0,194113 & 5,47 & 0,171847 \\
Q3 & 5,89 & 0,239824 & 5,89 & 0,186697 & 6,19 & 0,184707 \\
Máximo & 7,70 & 1,06 & 7,83 & 0,910164 & 7,91 & 0,779462 \\
\hline
\end{tabular}

Fuente: cálculos propios a partir del PIB determinado para la investigación 
acumuló más del $50 \%$ del PIB departamental, fenómeno más acusado en Risaralda y Quindío que en Caldas.

EI PIB per cápita disminuyó entre 2009 y 2011 en 13 municipios, en un rango de 0,001 a 0,112.

Los municipios cuyo PIB per cápita los ubicó como los de más baja condición socioeconómica, se encuentran en los departamentos de Risaralda (dos) y Caldas (cuatro).

El municipio más pobre, Quinchía, y el más rico, Balboa, están en el departamento de Risaralda. Llama la atención que Quinchía es uno de los municipios en los que el PIB per cápita disminuyó durante todo el período.

Cabe anotar que la variación de estos indicadores debería medirse en períodos más prolongados. En tal sentido, los datos disponibles no permitían caracterizar la tendencia del PIB a largo plazo.

En el cuadro 2 se presentan el promedio y la desviación del nivel de necesidades básicas insatisfechas de los municipios del Eje Cafetero del 2009 al 2011

El valor del índice de necesidades básicas insatisfechas puede considerarse un indicador de pobreza crónica. Los municipios con menos necesidades básicas insatisfechas fueron: Manizales, Villamaría, Armenia, Dosquebradas, Pereira y Calarcá. En estos municipios, solamente 10 a $15 \%$ de la población presentaba al menos una necesidad básica insatisfecha.

Las localidades más pobres y con mayor nivel de necesidades básicas insatisfechas, fueron Pueblo Rico, Mistrató, Norcasia y Quinchía. En estos municipios entre el 33 y el $52 \%$ de la población presentaba al menos una necesidad básica insatisfecha. Los municipios en los que coincidía el alto nivel de necesidades básicas insatisfecha y un PIB per cápita bajo, fueron

Cuadro 2. Promedio y desviación del valor del nivel de necesidades básicas insatisfechas por cuartiles. Eje Cafetero, 2009-2011

\begin{tabular}{lcc}
\hline & \multicolumn{2}{c}{ 2009-2011 } \\
\cline { 2 - 3 } Cuartiles & Promedio & Desviación \\
\hline Q1 & 16,42 & 3,09 \\
Q2 & 22,79 & 0,76 \\
Q3 & 24,95 & 0,52 \\
Q4 & 33,40 & 5,70 \\
\hline
\end{tabular}

Fuente: cálculos propios a partir de información del DANE (2012)
Pueblo Rico y Quinchía en Risaralda, localidades tradicionalmente pobres que seguían siéndolo en el momento del estudio.

A pesar de sus altos niveles de ingreso, algunos municipios presentaban una alta proporción de pobreza crónica. En Victoria y Marmato, entre el 30 y el $36 \%$ de la población presentaba al menos una necesidad básica insatisfecha. Marmato registraba el PIB más alto de Caldas y su índice de necesidades básicas insatisfechas fue de 35,9\%. Balboa, con el más alto PIB per cápita de los 53 municipios, también presentaba un índice de necesidades básicas insatisfechas por encima del $75 \%$ de la distribución por cuartiles; esta coexistencia de un PIB alto en una población predominantemente pobre según su nivel de necesidades básicas insatisfechas, ratifica la importancia de elegir e interpretar con cuidado los indicadores.

Los municipios con mayores niveles de necesidades básicas insatisfechas pertenecían a los departamentos de Caldas (nueve) y Risaralda (cuatro). Los municipios con menor proporción de habitantes afectados por necesidades básicas insatisfechas correspondieron a Caldas (siete), Quindío (tres) y Risaralda (tres).

El nivel de necesidades básicas insatisfechas departamental fue similar en Caldas, Quindío y Risaralda, y en los tres departamentos se presentó con frecuencia uno menor al de la capital departamental y los municipios del área metropolitana respectiva (Manizales y Pereira), o de Calarcá en el caso del Quindío. Los demás municipios presentaron un nivel superior al promedio departamental. El de menor nivel fue Manizales, con $10 \%$, y el de mayor nivel fue Pueblo Rico en Risaralda, con $53 \%$. Es decir que en lo que se refiere a necesidades básicas insatisfechas hay una brecha socioeconómica de 1 a 5 entre los municipios de la región.

Por otra parte, en la mayoría de los municipios el componente con mayores porcentajes de participación en el nivel de necesidades básicas insatisfechas fue el de la dependencia económica, seguido por el hacinamiento crítico. En Caldas los porcentajes respectivos fueron de 10,5 y 6 ; en el Quindío, de 9,2 y 4,9\%, y en Risaralda, de 9,1 y $6,8 \%$.

Los promedios de las tasas ajustadas de mortalidad por cuartiles en el trienio se presentan en el cuadro 3 y la variación de las tasas de mortalidad, en el cuadro 4. 
Cuadro 3. Promedio y desviación de las tasas de mortalidad ajustadas por edad, por enfermedad y por año, Eje Cafetero, 2009-2011

\begin{tabular}{lrrrrrr}
\hline \multirow{2}{*}{ Cuartiles } & \multicolumn{2}{c}{2009} & \multicolumn{2}{c}{$\mathbf{2 0 1 0}$} & \multicolumn{2}{c}{$\mathbf{2 0 1 1}$} \\
\cline { 2 - 7 } & Promedio & Desviación & Promedio & Desviación & Promedio & Desviación \\
\hline Enfermedades hipertensivas & & & & & & \\
Q1 & 3,42 & 1,18 & 6,11 & 1,96 & 5,26 & 1,76 \\
Q2 & 9,15 & 1,62 & 11,37 & 0,69 & 10,01 & 1,07 \\
Q3 & 14,33 & 1,38 & 15,95 & 1,54 & 13,73 & 1,62 \\
Q4 & 40,50 & 15,01 & 34,59 & 10,97 & 29,13 & 11,69 \\
Enfermedades isquémicas & & & & & \\
Q1 & 40,89 & 11,46 & 39,02 & 15,15 & 34,05 & 9,71 \\
Q2 & 68,61 & 5,61 & 78,56 & 5,10 & 64,36 & 5,39 \\
Q3 & 88,38 & 5,42 & 98,39 & 7,39 & 95,88 & 7,48 \\
Q4 & 142,41 & 30,31 & 147,80 & 29,91 & 133,35 & 24,23 \\
Enfermedades cerebrovasculares & & & & & \\
Q1 & 10,28 & 1,87 & 13,17 & 2,88 & 9,68 & 2,79 \\
Q2 & 20,55 & 2,46 & 23,45 & 1,71 & 19,31 & 2,14 \\
Q3 & 30,00 & 2,26 & 30,43 & 1,47 & 25,91 & 1,36 \\
Q4 & 42,51 & 6,89 & 43,33 & 9,44 & 39,99 & 12,65 \\
\hline
\end{tabular}

Fuente: cálculos propios a partir de los registros de defunción del DANE (2013)

Lo primero que llama la atención al revisar la distribución de las tasas es que, a pesar del ajuste por edad y el análisis por procedencia, se presentó una gran variabilidad. Esto hace pensar en condiciones que afectan la calidad de la clasificación de la causa, de la cobertura del registro o de ambas; sin embargo, los datos disponibles no permitieron aclarar este aspecto.

La distribución de las tasas ajustadas por cuartiles revelaron patrones irregulares, aunque en los tres grupos de causas predominó la tendencia al descenso, particularmente en las localidades con mayores riesgos.

Las tasas más altas correspondieron al subgrupo de muertes por enfermedades isquémicas; en todas las tasas de mortalidad se registró un incremento en 2010, y varias disminuciones en 2011.

\section{Desigualdades en la mortalidad por enfermedades cardiovasculares en los 53 municipios del Eje Cafetero}

Desigualdades en la mortalidad por enfermedades cardiovasculares según el PIB. El cuadro 5 permite visualizar los índices de las desigualdades en la mortalidad según el PIB. Su descripción e interpretación se hicieron por enfermedades, no obstante, se tuvo en cuenta que cada grupo de índices tiene fundamentos diferentes.

Las comparaciones de la mortalidad asociada con el PIB per cápita mediante mediciones de rango, permitieron valorar la amplitud de la brecha entre el municipio con el peor PIB y el municipio con el más alto. Durante el periodo de estudio el municipio en peores condiciones según el PIB per cápita fue Quinchía y, los mejores, Buenavista en 2009 y 2010, y Marmato en 2011.

Con respecto a las muertes por hipertensión arterial sistémica, no se identificó un patrón fijo de desigualdad asociado con el PIB. El riesgo de muerte por hipertensión arterial en Quinchía fue menor en 2009 y 2010 que en el municipio más rico, pero en 2011 esta condición se invirtió.

En la mortalidad por enfermedades isquémicas, en cambio, sí se encontró una brecha asociada repetidamente con el PIB; en los tres años analizados el riesgo de morir por esta causa fue mayor en el municipio con menor PIB (Quinchía) que en el municipio con el mejor PIB.

El análisis de la mortalidad por enfermedades cerebrovasculares también sugiere una brecha, pero en sentido inverso, a favor del municipio más pobre. El hecho de que los municipios con PIB más altos presentaran más riesgo de morir por enfermedades cerebrovasculares, podría obedecer a una mayor esperanza de vida y a un mejor registro; pero también, podría reflejar problemas de mal diagnóstico y subregistro en los municipios más pobres, donde la causa de muerte queda enmascarada. Este tipo de paradojas se ha descrito ya en epidemiología, sin embargo, los datos utilizados en el presente estudio no permitieron aclarar este punto. 
Cuadro 4. Variación anual y total de las tasas de mortalidad ajustadas por edad, por enfermedad, y por año según el producto interno bruto per cápita, Eje Cafetero, 2009-2011

\begin{tabular}{|c|c|c|c|c|c|c|}
\hline \multirow[t]{2}{*}{ Cuartiles } & \multicolumn{3}{|c|}{ Años } & \multirow{2}{*}{$\begin{array}{c}\text { Variación (\%) } \\
\text { 2009-2010 }\end{array}$} & \multirow{2}{*}{$\begin{array}{c}\text { Variación (\%) } \\
\text { 2010-2011 }\end{array}$} & \multirow{2}{*}{$\begin{array}{c}\text { Variación (\%) } \\
2009-2011\end{array}$} \\
\hline & 2009 & 2010 & 2011 & & & \\
\hline \multicolumn{7}{|c|}{ Enfermedades hipertensivas } \\
\hline Q1 & 3,42 & 6,11 & 5,26 & 79 & -14 & 54 \\
\hline Q2 & 9,15 & 11,37 & 10,01 & 24 & -12 & 9 \\
\hline Q3 & 14,33 & 15,95 & 13,73 & 11 & -14 & -4 \\
\hline Q4 & 40,50 & 34,59 & 29,13 & -15 & -16 & -28 \\
\hline \multicolumn{7}{|c|}{ Enfermedades isquémicas } \\
\hline Q1 & 40,89 & 39,02 & 34,05 & $-4,6$ & -13 & -17 \\
\hline Q2 & 68,61 & 78,56 & 64,36 & 14,5 & -18 & -6 \\
\hline Q3 & 88,38 & 98,39 & 95,88 & 11,3 & -3 & 8 \\
\hline Q4 & 142,41 & 147,8 & 133,35 & 3,8 & -10 & -6 \\
\hline \multicolumn{7}{|c|}{ Enfermedades cerebrovasculares } \\
\hline Q1 & 10,28 & 13,17 & 9,68 & 0,28 & -26 & -6 \\
\hline Q2 & 20,55 & 23,45 & 19,31 & 0,14 & -18 & -6 \\
\hline Q3 & 30,00 & 30,43 & 25,91 & 0,01 & -15 & -14 \\
\hline Q4 & 42,51 & 43,33 & 39,99 & 0,02 & -8 & -6 \\
\hline
\end{tabular}

Fuente: cálculos propios a partir de los registros de defunción del DANE (2013)

Cuadro 5. Desigualdades en la mortalidad por enfermedades cardiovasculares en los municipios del Eje Cafetero, 2009-2011

\begin{tabular}{|c|c|c|c|c|}
\hline \multirow[t]{2}{*}{ Enfermedades } & \multirow[t]{2}{*}{ Índices } & \multicolumn{3}{|c|}{ PIB per cápita } \\
\hline & & 2009 & 2010 & 2011 \\
\hline \multicolumn{5}{|c|}{ Índices de desigualdad basados en rangos } \\
\hline \multirow[t]{2}{*}{ Enfermedades hipertensivas } & Cociente de tasas & 0,51 & 0,35 & 1,33 \\
\hline & Diferencia de tasas & $-12,06$ & $-31,87$ & 3,83 \\
\hline \multirow[t]{2}{*}{ Enfermedades isquémicas } & Cociente de tasas & 3,36 & 2,69 & 3,04 \\
\hline & Diferencia de tasas & 58,22 & 61,38 & 75,21 \\
\hline \multirow[t]{2}{*}{ Enfermedades cerebrovasculares } & Cociente de tasas & 0,39 & 0,50 & 0,83 \\
\hline & Diferencia de tasas & $-14,89$ & $-12,27$ & $-4,21$ \\
\hline \multicolumn{5}{|l|}{ Índices de desproporcionalidad } \\
\hline \multirow[t]{2}{*}{ Enfermedades hipertensivas } & IRD & 0,61 & 0,50 & 0,04 \\
\hline & IRD acotado & 1,86 & 1,66 & 1,04 \\
\hline \multirow[t]{2}{*}{ Enfermedades isquémicas } & IRD & 2,39 & 0,13 & 0,19 \\
\hline & IRD acotado & $-11,26$ & 1,14 & 1,20 \\
\hline \multirow[t]{2}{*}{ Enfermedades cerebrovasculares } & IRD & 0,01 & $-0,28$ & $-0,11$ \\
\hline & IRD acotado & 1,01 & 0,76 & 0,89 \\
\hline \multicolumn{5}{|l|}{ Índices de concentración } \\
\hline \multirow[t]{2}{*}{ Enfermedades hipertensivas } & Concentración & $-0,10$ & $-0,08$ & $-0,01$ \\
\hline & Índice de Theil & 0,37 & 0,32 & 0,38 \\
\hline \multirow[t]{2}{*}{ Enfermedades isquémicas } & Concentración & $-0,39$ & $-0,02$ & $-0,03$ \\
\hline & Índice de Theil & 0,11 & 0,42 & 0,41 \\
\hline \multirow{2}{*}{ Enfermedades cerebrovasculares } & Concentración & 0,00 & 0,05 & 0,02 \\
\hline & Índice de Theil & 0,42 & 0,42 & 0,41 \\
\hline
\end{tabular}

IRD: índice relativo de desigualdad

Fuente: cálculos propios a partir de datos de la investigación y del registro de mortalidad del DANE (2012)

Desigualdades basadas en índices de regresión (índice relativo de desigualdad e índice relativo de desigualdad acotado). Dada la gran dispersión de los datos disponibles tanto para la mortalidad como para la medición de la pobreza, los análisis de regresión bivariada de los trastornos cardiovasculares y el PIB per cápita presentaron una variabilidad amplia en cada uno de los años analizados. En estas condiciones, el índice relativo de desigualdad acotado, que toma los promedios del mejor y el peor grupo, podría ser un mejor estimador de la desigualdad que el índice relativo de desigualdad crudo; este índice relativo de desigualdad acotado puede interpretarse de forma similar a como se interpretan los cocientes de rangos, pero en este caso, los cálculos no se 
limitaron a las localidades en condiciones socioeconómicas extremas porque se tuvieron en cuenta los demás municipios.

En 2009, los valores del índice relativo de desigualdad acotado de la mortalidad por enfermedades hipertensivas, isquémicas y cerebrovasculares, sugieren que los riesgos de morir por estas causas eran mayores en los municipios más pobres (con menor PIB per cápita). Este patrón de mayor riesgo de morir entre los más pobres, se mantuvo en 2010 y 2011 para las defunciones por enfermedades hipertensivas e isquémicas, pero no para las muertes por causas cerebrovasculares. En este último grupo de causas, el patrón de desigualdad asociado con el PIB fue menos claro; en 2009, el riesgo fue casi igual en municipios pobres y ricos $(1,01)$ y en los dos años siguientes el indicador se invirtió, lo que sugiere un mayor riesgo en los municipios más ricos.

Desigualdades basadas en los índices de concentración y de Theil. Los valores del índice de concentración para el trienio, no sugieren desigualdades importantes en las tasas ajustadas de mortalidad debida a enfermedades cardiovasculares que pudieran atribuirse a la pobreza medida por el PIB per cápita. Aunque la mortalidad causada por las enfermedades hipertensivas e isquémicas fue ligeramente mayor en los municipios con menor PIB en los tres años, los valores se acercan, en términos generales, a la diagonal de la igualdad.

En la figura 1 se muestran las curvas de concentración de la mortalidad según el PIB per cápita, las cuales reflejaron la tendencia de las tasas a acercarse a la diagonal de igualdad, excepto la mortalidad por enfermedades isquémicas en el 2009, año en que el índice fue de -0,39, lo que sugiere que la mortalidad por esta enfermedad tendió a concentrarse en los municipios con el peor PIB per cápita.

El cuanto al índice de Theil, los valores encontrados en el Eje Cafetero oscilaron entre 0,11 y 0,42; estos valores reflejan la proporción de defunciones que debe redistribuirse para alcanzar la igualdad.

Desigualdades en la mortalidad por enfermedades cardiovasculares medidas con base en las necesidades básicas insatisfechas. El análisis de desigualdades se repitió con las necesidades básicas insatisfechas, un indicador indirecto de pobreza que los analistas consideran más estable. Los resultados de las mediciones basadas en rangos, en la regresión y los índices, se registran en el cuadro 6, en el cual se presentan los resultados de la comparación entre los municipios con menor y mayor pobreza según sus necesidades básicas insatisfechas. El cociente de tasas extremas no evidenció un patrón claro de asociación entre las muertes por enfermedades cardiovasculares y la pobreza estructural reflejada en el nivel de necesidades básicas insatisfechas. El análisis basado en el cociente ponderado de tasas extremas, el cual se pondera según el tamaño de las poblaciones, sugiere un mayor riesgo de morir por enfermedades cardiovasculares en los municipios más pobres en los tres grupos de causas.

Según dicho nivel, el municipio con mayor pobreza fue Pueblo Rico, donde el 52,91 \% de la población tiene al menos una necesidad básica insatisfecha, y el de menor pobreza fue Manizales, donde ese porcentaje fue de $10,03 \%$.

El análisis de la mortalidad mediante esta medición demostró una variabilidad menor a la observada con el PIB per cápita; para las comparaciones, no obstante, se siguió utilizando el índice relativo de desigualdad acotado.

Entre los municipios con menor pobreza estructural figuraron: Manizales (Caldas), Villamaría (Caldas), Calarcá (Quindío), Circasia (Quindío), Chinchiná (Caldas), Filandia (Quindío), Salamina (Caldas), Anserma (Caldas) y Santa Rosa de Cabal (Risaralda). Las tasas en este grupo de municipios oscilaron entre 26,27 muertes por 100.000 habitantes en Manizales, más de 30 muertes en los municipios de Pereira, Calarcá y Armenia en el 2011, y hasta más de 60 en Salamina. Cabe señalar que algunos de estos municipios se encuentran en las áreas metropolitanas de los departamentos de Caldas, Quindío y Risaralda, o están cercanos a ellas.

Desigualdades en la mortalidad basadas en la regresión. Los valores del índice relativo de desigualdad acotado coincidieron con el análisis de rangos y sugieren que los municipios más pobres presentaron un menor riesgo de morir por enfermedades cerebrovasculares pero un mayor riesgo de morir por causas hipertensivas e isquémicas.

Desigualdades en la mortalidad según el índice de concentración y el de Theil. Los valores del índice de concentración estimados para los tres años, no sugieren desigualdades acentuadas en las tasas ajustadas de la mortalidad por enfermedades 


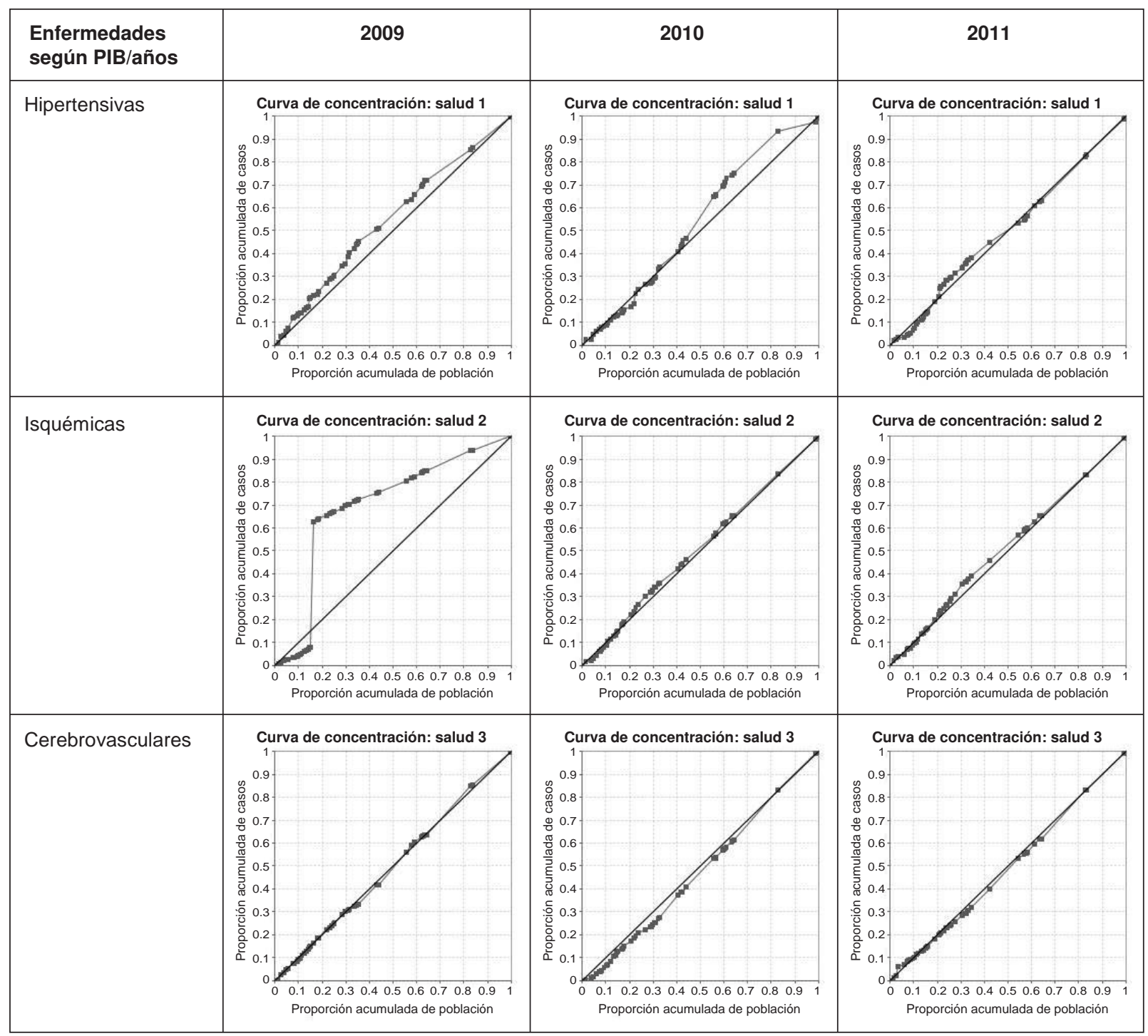

Fuente: resultados del análisis en el programa EPIDAT 3.1

Figura 1. Curvas de concentración de la mortalidad según el PIB en 53 municipios del Eje Cafetero, 2009-2011

cardiovasculares que pudieran atribuirse a la pobreza estructural medida con base en las necesidades básicas insatisfechas. Aunque, en general, los datos se acercaron a la diagonal de igualdad, las tasas de mortalidad por enfermedades hipertensivas e isquémicas fueron ligeramente mayores en los municipios con más necesidades básicas insatisfechas, sin que esa variable marcara grandes diferencias en el riesgo. La curva de concentración mostró, como se mencionó, la cercanía de los datos a la diagonal de igualdad, lo que sugiere poca incidencia de la desigualdad social en la mortalidad por enfermedades cardiovasculares medidas con base en dicho nivel (figura 2).
Los valores del índice de Theil transformado según la equivalencia de la entropía, oscilaron entre 0,11 y 0,42 , lo que sugiere el potencial de redistribución de la mortalidad que debiera lograrse para alcanzar el valor de plena igualdad. El índice de Theil mide la discrepancia entre la distribución de las personas y la distribución de la carga de enfermedad. Cuanto mayor es, mayor es la desproporción, pero sin que sea posible atribuirla a la condición socioeconómica más o menos favorable.

\section{Discusión}

La relación entre las desigualdades en la mortalidad por enfermedades cardiovasculares y las variables 
Cuadro 6. Desigualdades en la mortalidad por enfermedades cardiovasculares según el nivel de necesidades básicas insatisfechas en los municipios del Eje Cafetero, 2009-2011

\begin{tabular}{|c|c|c|c|c|}
\hline \multirow[t]{2}{*}{ Enfermedades } & \multirow[t]{2}{*}{ Índices } & \multicolumn{3}{|c|}{ Necesidades básicas insatisfechas } \\
\hline & & 2009 & 2010 & 2011 \\
\hline \multicolumn{5}{|c|}{ Índices de desigualdad basados en rangos } \\
\hline \multirow[t]{3}{*}{ Enfermedades hipertensivas } & Cociente de tasas & 1,61 & 6,53 & 1,51 \\
\hline & Diferencia de tasas & 6,15 & 13,72 & 5,46 \\
\hline & Cociente ponderado de tasas extremas & 0,05 & 0,21 & 0,05 \\
\hline \multirow[t]{3}{*}{ Enfermedades isquémicas } & Cociente de tasas & 0,54 & 1,43 & 1,26 \\
\hline & Diferencia de tasas & $-28,11$ & 33,0 & 20,60 \\
\hline & Cociente ponderado de tasas extremas & 0,02 & 0,05 & 0,04 \\
\hline \multirow[t]{3}{*}{ Enfermedades cerebrovasculares } & Cociente de tasas & 0,35 & 1,15 & 1,30 \\
\hline & Diferencia de tasas & $-16,96$ & 4,42 & 7,96 \\
\hline & Cociente ponderado de tasas extremas & 0,01 & 0,04 & 0,04 \\
\hline \multicolumn{5}{|l|}{ Índices de desproporcionalidad } \\
\hline \multirow[t]{2}{*}{ Enfermedades hipertensivas } & IRD & 0,73 & 0,90 & 0,49 \\
\hline & IRD acotado & 1,96 & 2,32 & 1,56 \\
\hline \multirow[t]{2}{*}{ Enfermedades isquémicas } & IRD & 1,14 & 0,30 & 0,13 \\
\hline & IRD acotado & 3,01 & 1,31 & 1,12 \\
\hline \multirow[t]{2}{*}{ Enfermedades cerebrovasculares } & IRD & $-0,13$ & $-0,26$ & $-0,13$ \\
\hline & IRD acotado & 0,89 & 0,78 & 0,89 \\
\hline \multicolumn{5}{|l|}{ Índices de concentración } \\
\hline \multirow[t]{2}{*}{ Enfermedades hipertensivas } & Concentración & $-0,12$ & $-0,15$ & $-0,08$ \\
\hline & Índice de Theil & 0,37 & 0,32 & 0,38 \\
\hline \multirow[t]{2}{*}{ Enfermedades isquémicas } & Concentración & $-0,19$ & $-0,05$ & $-0,02$ \\
\hline & Índice de Theil & 0,11 & 0,42 & 0,42 \\
\hline \multirow{2}{*}{ Enfermedades cerebrovasculares } & Concentración & 0,02 & 0,04 & 0,02 \\
\hline & Índice de Theil & 0,42 & 0,42 & 0,41 \\
\hline
\end{tabular}

IRD: índice relativo de desigualdad

Fuente: cálculos propios a partir de datos de la investigación y del registro de mortalidad del DANE (2012)

socioeconómicas, ha sido reconocida ampliamente; tal es el caso de los estudios realizados en países europeos (18) y en Finlandia (19). Sin embargo, en algunos de ellos la perspectiva del análisis ha sido establecer el vínculo entre las variables socioeconómicas y los factores de riesgo de enfermedad cardiovascular, lo cual limita la comprensión de las desigualdades socioeconómicas por sí mismas como fundamento explicativo de dicha mortalidad. En un estudio de Brasil (20) se demostró más claramente esta relación, puesto que la investigación se realizó con indicadores de pobreza. Se destacan en la literatura científica reciente los análisis de las desigualdades en Andalucía, los cuales se llevaron a cabo con base en una propuesta amplia de indicadores relacionados con factores determinantes sociales (21). Los resultados del estudio en el Eje Cafetero, aunque no son comparables con los estudios mencionados, revelan la complejidad de la relación entre las desigualdades socioeconómicas y la mortalidad por enfermedades isquémicas e hipertensivas, ya que las carencias que evidenciaron los valores de necesidades básicas insatisfechas superaron las interpretaciones basadas en la consideración de lo socioeconómico como un factor de riesgo más o como un factor de riesgo que subyace a los estilos de vida y los comportamientos.

Lo señalado coincide con los resultados del estudio, ya que el riesgo de morir por enfermedad isquémica e hipertensiva fue más alto en los municipios con más necesidades básicas insatisfechas. La mortalidad por enfermedad hipertensiva tendió a concentrarse en los municipios con un mayor nivel de tales necesidades.

Con relación al PIB, se encontraron más muertes por enfermedad hipertensiva en los municipios con menor PIB per cápita en los años 2009 y 2010, y por enfermedad isquémica en los años 2010 y 2011. No obstante, deben tenerse en cuenta las limitaciones de este indicador en la medición de las desigualdades, como se señala más adelante.

Conviene mencionar que otras perspectivas más completas se abren paso e intentan superar el modelo de factores de riesgo; se trata del análisis del efecto de las malas condiciones socioeconómicas a lo largo del curso de la vida de las poblaciones. Algunos de estos análisis pretenden dar cuenta del gradiente generado por las condiciones 


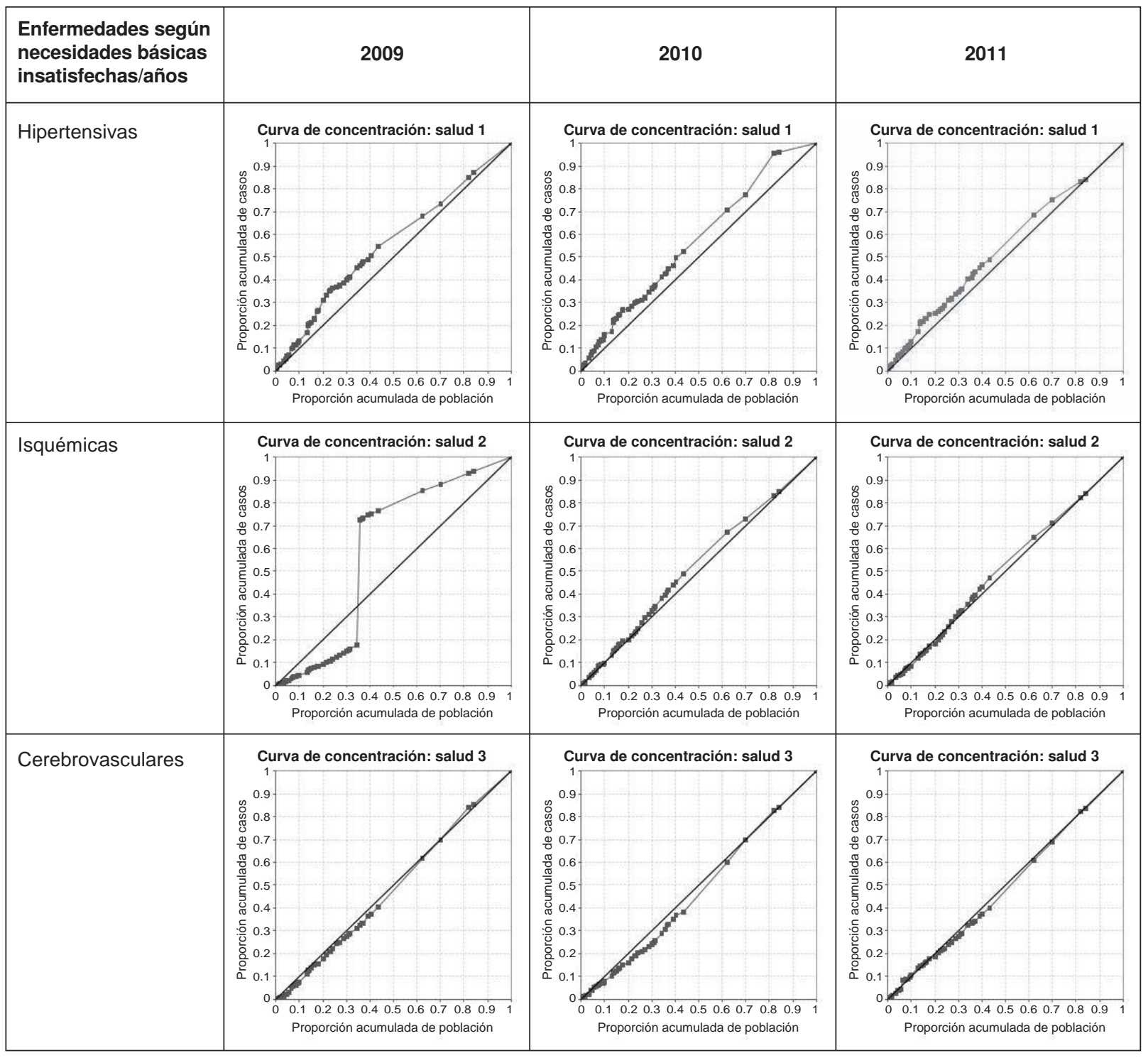

Fuente: resultados EPIDAT 3.1

Figura 2. Curvas de concentración de la mortalidad según el nivel de necesidades básicas insatisfechas en 53 municipios del Eje Cafetero, 2009-2011

socioeconómicas y su relación con la mortalidad $(22,23)$. De particular interés es la evaluación de los modelos de análisis del curso de la vida y sus efectos sobre el sistema cardiovascular (24), los cuales sustentan el impacto de las experiencias negativas debidas al nivel socioeconómico en los efectos cardiovasculares.

Una conclusión del presente trabajo se refiere a la importancia del análisis de las desigualdades en zonas geográficas específicas debida a factores contextuales que pueden explicar los resultados en salud, más allá de los factores individuales. Además, la caracterización de zonas geográficas con los peores indicadores socioeconómicos y de salud, facilita el diseño de políticas e intervenciones integrales coordinadas entre diversos sectores.

Las limitaciones identificadas durante la ejecución de la investigación pueden agruparse en tres grupos: las fuentes de información, los indicadores socioeconómicos y las medidas utilizadas.

Inicialmente, el estudio se propuso medir las desigualdades en la morbilidad y la mortalidad por enfermedades hipertensivas, isquémicas y cerebrovasculares en 53 municipios del Eje Cafetero. La fuente más apropiada para conocer la morbilidad 
son los estudios de población y, por lo general, en los municipios no se hacen este tipo de estudios. Aunque el país cuenta con datos de morbilidad sentida recogidos por la Encuesta Nacional de Salud 2007, no existe información desagregada de esta variable a nivel municipal. Ante la ausencia de estudios epidemiológicos de población, surgió la opción alternativa de usar los registros individuales de prestación de servicios como fuentes de información de la morbilidad atendida o registrada; los informes de la morbilidad registrada reflejan fundamentalmente la estructura de la oferta y el uso de los servicios de salud por parte de la población, pero no dan cuenta de la morbilidad de la población que no acude a los servicios de salud. Este aspecto es objeto de discusión en el Análisis de la Situación de Salud, ASIS III, 2010, cuando se señala que al analizar los registros se puede subestimar la morbilidad. De todos modos, se revisó la calidad de los registros de la morbilidad atendida, pero se consideró que presentaban serias limitaciones y se optó por descartar esta información.

En consecuencia, se decidió utilizar la información de la mortalidad registrada, asumiendo que podía reflejar mejor la dinámica epidemiológica de las enfermedades cardiovasculares en la región. Aunque la calidad de estos registros ha mejorado en el país, la revisión de la causa básica de defunción por subgrupos de enfermedades cardiovasculares, puso en evidencia variaciones importantes de un año a otro en los municipios, lo que reflejaría los problemas de clasificación y diferenciación entre las categorías.

EI PIB per cápita calculado específicamente para cada municipio, mide la renta media de las personas en el grupo y se ha considerado como una de las mediciones directas de pobreza y la primera en ser usada con relativa frecuencia, según lo señalado por Caballero en el balance que hace de la información disponible sobre pobreza y desigualdad (13). Sin embargo, actúa como una medida de resumen y en tal sentido no mide la brecha entre pobres ni la distribución del ingreso. Un municipio con una alta concentración de la riqueza puede presentar un PIB per cápita alto aunque la mayoría de la población sea pobre. A lo anterior se suma que el PIB per cápita de los departamentos de Caldas, Quindío y Risaralda tiende a acercarse al promedio nacional y que, de alguna manera, refleja una reducción de las desigualdades en comparación con el país (25).
Un tercer elemento que merece destacarse es la coexistencia de un PIB per cápita alto con proporciones elevadas de población en condiciones de pobreza crónica (alto nivel de necesidades básicas insatisfechas); en la región estudiada, nueve municipios con un PIB per cápita que superaba el tercer cuartil presentaron los más altos de dichos niveles. En estos casos, a pesar de tener un PIB per cápita elevado, la población de un municipio puede presentar situaciones que contribuyen a la precariedad de sus condiciones de vida y a la exclusión social, como son el bajo nivel educativo o la pertenencia a minorías étnicas; tal es el caso de Marmato en Caldas y de Buenavista en Quindío, que registraron reiteradamente mediciones compatibles con el efecto de la desigualdad social en la mortalidad por enfermedades cardiovasculares.

El campo de evaluación de las necesidades básicas insatisfechas es el de bienes y servicios requeridos para su satisfacción, lo que permite clasificar a la población según su acceso a los servicios sanitarios, las condiciones de su vivienda, la dependencia económica, la inasistencia escolar y el hacinamiento. En cada departamento, dos a tres municipios presentaron altos niveles de necesidades básicas insatisfechas, y alrededor del $13 \%$ de ellos registraron niveles entre 30 y $53 \%$.

Cabe señalar que los municipios con mayor nivel de necesidades básicas insatisfechas, como Pueblo Rico, presentan desde antes de 1993 la mayor proporción de personas afectadas en el departamento de Risaralda, superando ampliamente el promedio nacional. Igualmente, en los municipios de Pueblo Rico y Quinchía se reportó una evolución desfavorable de las condiciones de vida, reflejada en la tendencia decreciente en el aumento del ingreso per cápita, el retroceso en la equidad distributiva del ingreso y un menor crecimiento del índice de condiciones de vida en el período entre los censos (26). Así, en Quinchía se registró una disminución del PIB per cápita y, en Pueblo Rico, un PIB que disminuía o no cambiaba.

Pese a las limitaciones mencionadas, los resultados de las mediciones de la desigualdad evidenciaron un mejor desempeño; es posible que otros indicadores, como la calidad de vida, el desarrollo humano, o la vulnerabilidad, permitan discriminar más finamente las poblaciones con desigualdades sociales en salud.

En relación con los indicadores de desigualdad encontrados, se observaron contradicciones entre aquellos basados en rangos y los basados en la 
regresión. Si bien la lógica que subyace a cada uno de estos es diferente, es relevante tener en cuenta que los primeros pasan por alto lo que sucede en los grupos intermedios y que, además, la falta de linealidad entre el indicador socioeconómico y el de salud incide en los resultados. Los segundos, es decir, los indicadores basados en la regresión (índice relativo de desigualdad acotado en Epidat), mostraron mejor la desigualdad. Los IRD acotados evidenciaron la relación entre la mortalidad por enfermedades hipertensivas $e$ isquémicas, $y$ un menor PIB y un mayor nivel de necesidades básicas insatisfechas en los municipios.

\section{Agradecimientos}

A los funcionarios del Programa de Investigación en Salud de Colciencias, por el apoyo otorgado a los investigadores durante el proyecto.

\section{Conflicto de intereses}

Los autores expresan que no existen conflictos de intereses.

\section{Financiación}

Proyecto (código 1259-519-28192) financiado por Colciencias a través de la convocatoria 519 de 2010.

\section{Referencias}

1. Di Cesare M. El perfil epidemiológico de América Latina y el Caribe: desafíos, límites y acciones. Comisión Económica para América Latina y el Caribe (CEPAL). Santiago de Chile: Naciones Unidas; 2011. Fecha de consulta: 21 de agosto de 2014. Disponible: http://www.cepal.org/publicaciones/ xml/9/44309/lcw395.pdf.

2. Organización Panamericana de la Salud, Organización Mundial de la Salud. Salud en las Américas. Panorama regional y perfiles de país. 2012. Fecha de consulta: 21 de agosto de 2014. Disponible en: http://www.paho.org/ saludenlasamericas/index.php?option=com_content\&view $=$ article $\&$ id $=9 \% 3$ Aedicion-2012\&catid=9\%3Apublication\&lt emid=14\&lang=es.

3. Ministerio de Salud, Facultad Nacional de Salud Pública de la Universidad de Antioquia. Análisis de la situación de salud en Colombia 2002-2007. Bogotá: Imprenta Nacional de Colombia; 2011. Fecha de consulta: 21 de agosto de 2014. Disponible en: http://www.minsalud.gov.co/ Documentos\%20y\%20Publicaciones/ASIS-Tomo\%20III.

4. Ministerio de Salud y Protección Social. Plan Decenal de Salud Pública. PDSP, 2012-2021. Bogotá: Imprenta Nacional de Colombia; 2013

5. Observatorio Nacional de Salud-Instituto Nacional de Salud. Enfermedad cardiovascular: principal causa de muerte en Colombia. Boletín № 1. Diciembre de 2013. Fecha de consulta: 22 de agosto de 2014. Disponible en: http://www.ins.gov.co/lineas-de-accion/ons/boletin\%201/ boletin_web_ONS/boletin_01_ONS.pdf.
6. Rodríguez J, Acosta N, Peñaloza R. Carga de enfermedad en Colombia. Resultados alcanzados. Centro de Proyectos para el Desarrollo (CENDEX). Bogotá, D.C.: Pontificia Universidad Javeriana; 2008. Fecha de consulta: 21 de agosto de 2014. Disponible en: http://www.cendex.org.co/ GPES/informes/PresentacionCarga_Informe.pdf.

7. Ministerio de la Protección Social. Plan Nacional de Salud Pública 2007-2010. Decreto № 3019. Fecha de consulta: 21 de agosto de 2014. Disponible en: http://new.paho. org/hq/dmdocuments/2010/Politicas_Nacionales_SaludColombia_2007-2010.pdf.

8. World Health Organization. Global status report on noncommunicable diseases 2011. Fecha de consulta: 25 de noviembre de 2011. Disponible en: http://www.who.int/nmh/ publications/ncd_report2010/es/.

9. Organización Panamericana de la Salud, Organización Mundial de la Salud. 28 $8^{\underline{a}}$ Conferencia Sanitaria Panamericana. Resolución csp28.r13 Estrategia para la prevención y el control de las enfermedades no transmisibles. Washington, D.C.: OPS, OMS: 2012. Fecha de consulta: 21 de agosto de 2014. Disponible en: http://www. paho.org/hq/index.php?option=com_docman\&task=doc_ view\&gid=19267\&ltemid.

10. World Health Organization. World Conference of Social Determinants in Health. Closing the Gap: Policy into practice on social determinants of health. Río de Janeiro; WHO; 2011. Fecha de consulta: 22 de agosto de 2014. Disponible en: http://www.who.int/sdhconference/Discussion-PaperEN.pdf.

11. Organización Mundial de la Salud. Cerrando la brecha: la política de acción sobre los determinantes sociales de la salud. Río de Janeiro; WHO; 2011. Fecha de consulta: 6 de abril 2015. Disponible en: http://www.who.int/sdhconference/ discussion_paper/Discussion-Paper-SP.pdf.

12. Ministerio de Salud, Universidad de Antioquia, Facultad Nacional de Salud Pública. Análisis de la situación de salud en Colombia 2002-2007. Análisis de las desigualdades e inequidades 2011. Fecha de consulta: 21 de agosto de 2014. Disponible en: http://www.minsalud.gov. co/Documentos\%20y\%20Publicaciones/ASIS-Tomo\%20 VI-An\%C3\%A1lisis\%20de\%20desigualdades $\% 20$ e\%20 inequidades.pdf.

13. Caballero C, García M, Vélez Z. Pobreza y desigualdad. Un balance de la información disponible. Fecha de consulta: 22 de agosto de 2014. Disponible en: http://www.colombialider. org/wp-content/uploads/2011/09/Pobreza-y-Desigualdad. pdf.

14. Feres JC, Mancero X. Necesidades básicas insatisfechas y su aplicación en América Latina. CEPAL estudios estadísticos y prospectivos. División de Estadística y Proyecciones Económicas. Santiago de Chile: CEPAL; 2001. Fecha de consulta: 22 de agosto de 2014. Disponible en: http://www. cepal.org/publicaciones/xml/4/6564/lcl1491e.pdf.

15. Schneider MC, Castillo-Salgado C, Bacallao J, Loyola E, Mujica O, Vidaurre M, et al. Métodos de medición de las desigualdades de salud. Rev Panam Salud Pública. 2002;12:394-414. http://dx.doi.org/10.1590/S102049892002001200006

16. Vallecilla J. La economía de los municipios de Caldas, Quindío, Risaralda 2007-2011. Regiones. 2013;8:85-106. 
17. Hervada-Vidal X, Santiago-Pérez MI, Vázquez-Fernández E, Castillo-Salgado C, Loyola-Elizondo E, Silva-Ayçaguer LC. EPIDAT 3.0 Programa para análisis epidemiológico de datos tabulados. Rev Esp Salud Pública. 2004;78:277-80.

18. Mackenbach JP, Stirbu I, Roskam AJ, Schaap MM, Menvielle G, Leinsalud M, et al. Socioeconomic inequalities in health in 22 European countries. N Engl J Med. 2008. 358:2468-81. http://dx.doi.org/10.1056/NEJMsa0707519

19. Lammintausta A. Effects of socioeconomic status and sociodemographic features on cardiovascular disease mortality and morbidity in Finland (thesis). Turun: University of Turku; 2013. Fecha de consulta: 30 de marzo de 2015. Disponible en: https://www.doria.fi/bitstream/ handle/10024/90256/AnnalesD1068Lammintausta. pdf? sequence $=2$.

20. Carneiro-de Oliveira D, Salgado-Barreria A, TaracidoTrunk M, Figueiras-Guzmán A. Efecto de las desigualdades socioeconómicas en la mortalidad de la ciudad de fortaleza, Ceará, Brasil, durante el año 2007. Rev Esp Salud Pública. 2010;84:441-50.

21. Pujolar A, Martínez MD, Daponte A. Primer informe sobre desigualdades y salud en Andalucía. Andalucía: Asociación para la Defensa de la Sanidad Pública de Andalucía. 2008. Fecha de consulta: 30 de marzo de 2015. Disponible en: http://web.ua.es/opps/docs/informes/INDESAN_final_.pdf.
22. Kamphuis C, Turrell G, Giskes K, Mackenbach JP, van Lenthe FJ. Socioeconomic inequalities in cardiovascular mortality and the role of childhood socioeconomic conditions and adulthood risk factors: A prospective cohort study with 17-years of follow up. BMC Public Health. 2012,12:1045. http://dx.doi.org/10.1186/1471-2458-12-1045

23. Galobardes B, Smith GD, Lynch JW. Systematic review of the influence of childhood socioeconomic circumstances on risk for cardiovascular disease in adulthood. Ann Epidemiol. 2006;16:91-104.

24. Pollitt RA, Rose KM, Kaufman JS. Evaluating the evidence for models of life course socioeconomic factors and cardiovascular outcomes: A systematic review. BMC Publ Health. 2005;5:7. http://dx.doi.org/10.1186/1471-2458-5-7

25. Galvis LA, Meisel A. Persistencia de las desigualdades regionales en Colombia: un análisis espacial. Cartagena: Banco de la República; 2010. Fecha de consulta: 23 de agosto de 2014. Disponible en: http://www.banrep.gov.co/ docum/Lectura_finanzas/pdf/DTSER-120.pdf.

26. Gaviria MA, Sierra HD. Risaralda: una mirada a las condiciones de vida de su población. Revista Académica e Institucional de la Universidad Católica Popular de Risaralda (UCPR). 2000. Edición No. 58. Fecha de consulta: septiembre 2 de 2014. Disponible en: http://www.ucp.edu. co/paginas/revista58/risaralda.htm. 